\title{
Countermeasure for Interrupted-sampling Repeater Jamming based on Fractional Fourier Transformation
}

\author{
Ming Liu ${ }^{\mathrm{a}}$, Tao Shu and Qingrong Chen \\ Air Force Engineering University, 710051 Xi'an, China
}

\begin{abstract}
Today, the interrupted-sampling repeater jamming (ISRJ) has posed a serious threat to LFM radars. In this paper, a countermeasure to suppressing ISRJ based on fractional Fourier transformation (FrFT) is presented. The echo signals, mixed with ISRJ, will be processed in FrFT of specific order, and then a narrow band filter based on parameters of FrFT is designed to extract the target signals and suppress interference signal, so as to realize the target detection. The three kinds of models of ISRJ (ISDRJ, ISRRJ, ISCRJ) are introduced; the anti-jamming principles are analyzed and simulated. The simulation results show that, the algorithm has low complexity and takes short operation time, and it is effective in low SNR or high JSR condition.
\end{abstract}

\section{INTRODUCTION}

Linear frequency modulation (LFM) signal is widely used in tracking radar. With the development of LFM interference method, jammers with digital radio frequency memory (DRFM) can accurately reproduce the received radar signals and simulate them, resulting in coherent jamming effect, especially for LFM signals. The development and perfection of interrupted-sampling repeater jamming (ISRJ) [1], which is flexible and has diverse forms, can form several false targets [2] to deceive radars, as well as creating the effect of noise suppression to a certain extent. With the flexibly change of repeating and modulation method, interference effect is improving, which has posed a serious threat to the tracking radar, so it is urgent to seek a way to counter this type of interference [3].

In recent years, the fractional Fourier transform (FrFT) in the field of radar signal processing applications has developed rapidly: Paper [4] introduces novel theorems for FRFT; the paper [5] proposed a unified focusing algorithm for several modes of SAR based on FrFT; literature [6] used FrFT for detecting targets in sea clutter; paper [7] put forward the analysis for FRFT-OFDM systems to frequency offset in time-frequency selective fading channels, [8] analysis of the intermittent sampling repeater jamming parameters influence on the performance of the interference.

This paper is based on eigenvalue decomposition discrete FrFT algorithm [9-10], isolates interference and target signal. By narrow band filtering, the jamming will be suppressed and the target echo will be retained. The simulation results verify the validity of this method.

\section{Jamming models}

\footnotetext{
a Corresponding author:929237749@qq.com
}

The interrupted-sampling repeater jamming (ISRJ) affect LFM radars mainly by intermittent sampling in radar pulse period and repeat sampling signals back according to certain rules. The jamming and target echo mix together, which takes severe effects on radars after the relevant treatment.

The linear frequency modulation (LFM) signal is in the form of:

$$
x_{r}(t)=\operatorname{rect}\left(\frac{t}{T}\right) e^{j 2 \pi f_{0} t+j \mu \pi t^{2}},|t|<\frac{T}{2}
$$

Where $T$ is radar pulse width; $\mu=B / T$ is chirp rate of LFM signal; $B$ is the signal bandwidth; $f_{0}$ is carrier frequency. A mathematical model of arbitrary pulse signal is:

$$
x_{n}(t)=\operatorname{rect}\left[\frac{t-(n-1) T_{J}}{\tau_{J}}\right] \cdot e^{j 2 \pi f_{0} t+j \pi \mu t^{2}}
$$

Where $T_{J}$ is the sampling repetition period of the interrupted sampling signal is the interference pulse width; $\tau_{J}$ is sampling pulse width. The signal obtained by reconnaissance sampling of the jammer is an accumulation of sub pulse signals:

$$
\left.x(t)=\sum_{n=1}^{N} \operatorname{rect}\left[\frac{t-(n-1) T_{J}}{\tau_{J}}\right)\right] \cdot e^{j 2 \pi f_{0} t+j \pi \mu t^{2}}
$$

The main interference modes are interrupted-sampling directed-repeater jamming (ISDRJ), repeated-repeater jamming (ISRRJ) and cyclic repeater jamming (ISCRJ).

\subsection{ISDRJ}

ISDRJ will forward a small sample of LFM signal immediately after sampling, and then sampling, 
processing, forwarding the next paragraph.

Fig. 1 shows time sequence of interference machine timing. The ISDRJ can be regarded as a bit of direct sampling signal, delayed after a sampling time, shifted frequency in frequency domain and forwarded. It can be expressed as:

$$
x_{J}(t)=x\left(t-\tau_{J}\right)
$$

where $\tau_{J}$ is sampling pulse width.

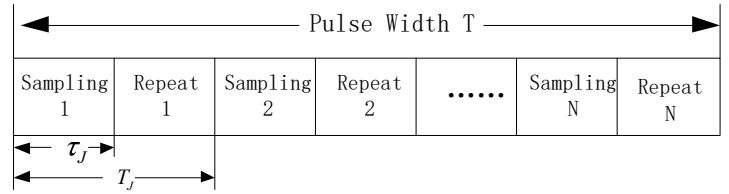

Figure 1. Time sequence of ISDRJ

\subsection{ISRRJ}

ISRRJ is similar to ISDRJ, but it only forwards the same sampling signal in intermittent period. The working sequence of the jammer is shown in Fig. 2.

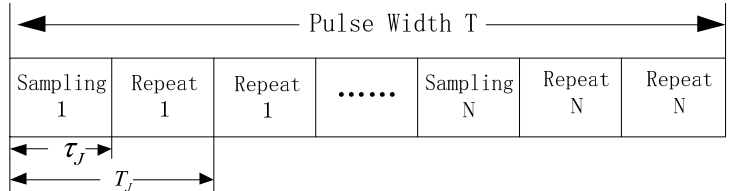

Figure 2. Time sequence of ISRRJ

The ISRRJ is in the form of:

$$
x_{J}(t)=\sum_{i=1}^{M} x\left(t-i \tau_{J}\right)
$$

Where $\mathrm{M}$ is the number of sampling pulses that can be forwarded during the sampling interval. The principle of ISRRJ is equivalent to delaying the sampling signal with time $k \tau_{J}, k=1,2 \cdots M$ several times and then forwarding.

\subsection{ISCRJ}

The ISCRJ moves the signals in the previous sampling periods in order and then forwards them. The working sequence of the jammer is shown in Fig.3.

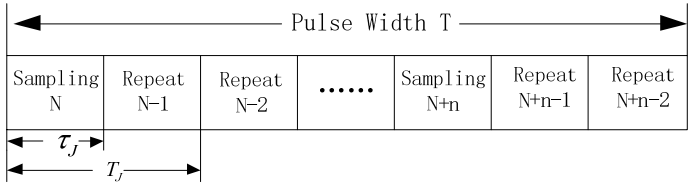

Figure 3. Time sequence of ISCRJ

The principle is equivalent to increasing the time delay of the interference signals by time $\tau_{J}-k\left(T_{J}+\tau_{J}\right) \quad$,where $k=0,2, \cdots R-1$ and $R=\min \{M, N\}$, obtained by sampling, and then forwarding them. Its mathematical expression is:

$$
x_{J}(t)=\sum_{i=0}^{R-1} x\left[t-\tau_{J}-i\left(T_{J}+\tau_{J}\right)\right]
$$

No matter what kind of repeating, they can all produce related interference and take obvious effect on LFM radars by matched filtering to generate the false targets of different locations. On certain conditions, by complex forwarding and modulation method, the effect of suppression can be generated. Comparison of interference performance in different modes is shown in Tab.1, where *** represent the best and $*$ is the worst.

Table 1. Comparison of interference effects

\begin{tabular}{|c|c|c|c|}
\hline $\begin{array}{c}\text { Jamming } \\
\text { modes }\end{array}$ & $\begin{array}{c}\text { Pulse width of } \\
\text { single sampling }\end{array}$ & $\begin{array}{c}\text { Deception } \\
\text { effect }\end{array}$ & $\begin{array}{l}\text { Suppression } \\
\text { effect }\end{array}$ \\
\hline ISDRJ & $\mathrm{B} / 2 \mathrm{~N}$ & $*$ & $* * *$ \\
\hline ISRRJ & $\mathrm{B} /((\mathrm{n}+1) \mathrm{N})$ & $* *$ & $* *$ \\
\hline ISCRJ & $\mathrm{B} /((\mathrm{n}+1) \mathrm{N})$ & $* * *$ & $*$ \\
\hline
\end{tabular}

\section{FrFT of LFM Signals}

Fractional Fourier transform (FrFT) is a generalized form of the traditional Fourier transform (FT), which combines time domain and frequency domain to analyze and process signals.

It can be expressed as:

$$
X_{\alpha}(u)=\int_{-\infty}^{\infty} x(t) K_{\alpha}(t, u) d t
$$

Where $K_{\alpha}(t, u)=$

$$
\left\{\begin{array}{lc}
\sqrt{1-j \cot \alpha} e^{j 2 \pi\left(t^{2}+u^{2} / 2\right) \cot \alpha-j 2 \pi u t \csc (\alpha)} & , \alpha \neq n \pi \\
\delta(t-u) & , \alpha=2 n \pi \\
\delta(t+u) & , \alpha=(2 n+1) \pi \\
e^{-j 2 \pi t u} & , \alpha=n(\pi / 2)
\end{array}\right.
$$

LFM signals exhibit different energy aggregation in different fractional domains, and their order of transformation $\alpha$ is determined by rotation angle. When satisfying $\mu+\cot \alpha=0$, the LFM signal generates energy aggregation in the corresponding transform domain.

The LFM signal amplitude after the optimum order FrFT is:

$$
\left|X_{\alpha_{o p t}}(u)\right|=\sqrt{B T} \frac{\sin (\pi B u)}{\pi B u}
$$

\section{Principle and Realization of Countermeasure}

When the radar signal is processed by quadrature phase detector, the echo with jamming can be expressed as:

$$
r(t)=x_{r}(t)+x_{J}(t)+n(t)
$$

Where $n(t)$ is the additive Gauss white noise. The formula (10) is transformed by FrFT:

$$
\begin{aligned}
R(u) & =F \alpha_{o p t}[r(t)] \\
& =X_{r \alpha_{o p t}}(u)+X_{J \alpha_{o p t}}(u)+N_{\alpha_{o p t}}(u)
\end{aligned}
$$

The echo signal is:

$$
\begin{aligned}
& X_{r \alpha_{o p t}}(u)= \sqrt{1-j \cot \alpha_{o p t}} \\
& \cdot T e^{j \pi u^{2} \cot \alpha_{o p t}} \frac{\sin (\pi B u)}{\pi B u}
\end{aligned}
$$

The FrFT of ISRJ is: 


$$
\begin{array}{r}
X_{\alpha_{o p t}}(u)=\mathrm{N} \cdot \sqrt{1-j \cot \alpha_{o p t}} e^{j \pi u^{2} \cot \alpha_{o p t}} \\
\cdot \tau_{J} \frac{\sin \left[\pi\left(u \csc \alpha_{o p t}\right) \tau_{J}\right]}{\pi\left(u \csc \alpha_{o p t}\right) \tau_{J}}
\end{array}
$$

According to the time shift characteristic of FrFT, the FrFT results of intermittent sampling and forward jamming signals are obtained

$$
\begin{aligned}
& X_{J \alpha_{o p t}}(u)=F_{\alpha_{o p t}}\left[x_{J}\left(t-\tau_{J}\right)\right] \\
& =k X_{\alpha_{o p t}}\left(u-\tau \cos \alpha_{o p t}\right)
\end{aligned}
$$

Where:

$$
k=e^{-j \tau^{2}\left(\sin \alpha_{o p t} \cos \alpha_{o p t}\right) / 2+j u \tau_{J} \sin \alpha_{o p t}}
$$
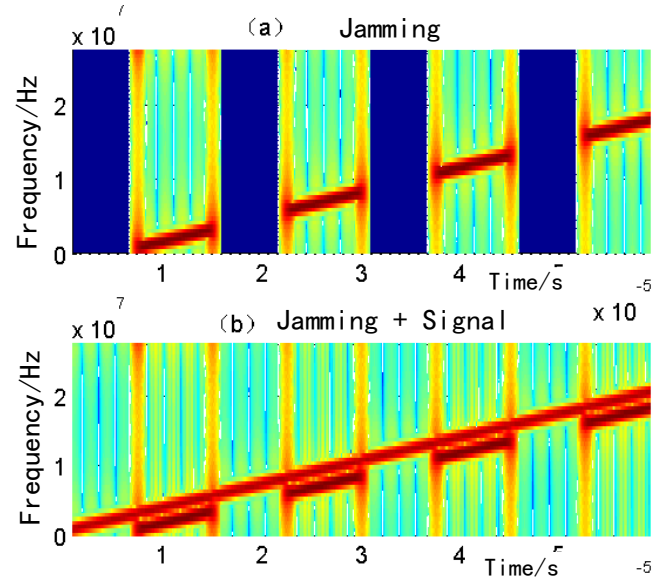

Figure 4. Time-frequency analysis of target echo and ISRJ

Fig.4 shows time-frequency analysis of target echo and ISRJ. The ISRJ FrFT results are closely related to sampling pulse width, repeating mode and the frequency shift, usually distribute as sections in the signal bandwidth (shown in Fig.4 (a)); because of lag in time domain, the ISRJ and target signals are easily distinguished (shown in Fig.4 (b)). The interference is different from the target signal in location and distribution, and it lags behind the target signal in the FrFT domain with $\tau_{J} \sin \alpha_{o p t}$.

For a cooperative LFM signal, the location after FrFT is determined, while the jamming and the target echo signal can be separated in the FrFT domain. With this feature, interference can be suppressed in the FrFT domain.

According to the above principle, the suppression of ISRJ by LFM radar can be carried out by FrFT (shown in Fig.5). The main process is as follows:

1. According to the LFM waveform data and the receiving window width, the optimum transform order is calculated.

$$
\alpha_{\text {opt }}=-\tan ^{-1}\left(F s^{2} / \mu L\right)
$$

Where $L$ is the length of the receiving window, and $F S$ is the sampling frequency.

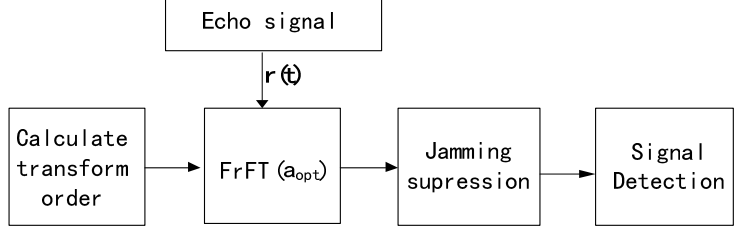

Figure 5. Process of ISRJ suppression

2. The FrFT result $R(u)$ is calculated according $\alpha_{\text {opt }}$ after quadrature phase demodulation .

3. Design a fractional order narrowband filter

$$
M(u)=\sum_{i=-w / 2}^{w / 2-1} \delta\left(u-\left(\frac{T^{2} f_{s}\left|\tan \left(\alpha_{o p t}\right)\right|}{2 B}+i\right)\right)
$$

While $w$ is the width of the pass band; $i$ is integer. Filter result is:

$$
\begin{aligned}
& Y(u)=R(u) M(u) \\
& =\left[X_{r \alpha_{o p t}}(u)+X_{J \alpha_{o p t}}(u)+N_{\alpha_{o p t}}(u)\right] M(u)
\end{aligned}
$$

After processing, the peak pulse echo in the FrFT domain aggregation will be retained, and the ISRJ will be suppressed because of the different peak position.

4. After filtering, the signal will be processed by CFAR detection and other follow-up measures.

\section{5 simulation results and analysis}

In this part, The simulation and analysis of the above anti-jamming methods will be carried out.

The simulation parameters are set as follows: LFM bandwidth $\mathrm{B}=4 \mathrm{MHz}$, pulse width $\mathrm{T}=60 \mathrm{~s}$, sampling frequency $\mathrm{Fs}_{\mathrm{s}}=28 \mathrm{MHz}$, the $\mathrm{FrFT}$ rotation angle is calculated as $\alpha_{\text {opt }}=-1.4290, \mathrm{SNR}=0 \mathrm{~dB}$.

\subsection{ISDRJ}

A radar pulse is sampled 4 times and repeated directly with 4 times. The sampling pulse duration is $7.5 \mu \mathrm{s}$, the sampling repetition period is $15 \mu \mathrm{s}$, and the jamming-signal ratio (JSR) is $6 \mathrm{~dB}$.

Fig.6 (a) shows a matched filtering result of ISRJ and target echo, and the jamming generates a number of false targets in the receiving window, which seriously interferes with radar detection. Fig. 6 (b) is the spectrum of the target echo and the interference. The frequency spectrum of the interference signal is segmented in the whole signal bandwidth, which is consistent with the previous analysis.
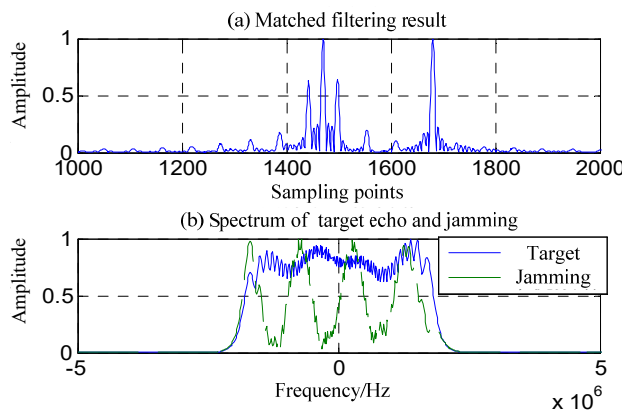

Figure 6. Target echo and jamming 
Using the anti-jamming method, the result is shown in Fig. 7. Fig. 7 (a) shows the FrFT result of the mixed signals, and Fig. 7 (b) is the result of interference suppression. After suppression, only the target signal is left. This method is effective to suppress the ISDRJ.
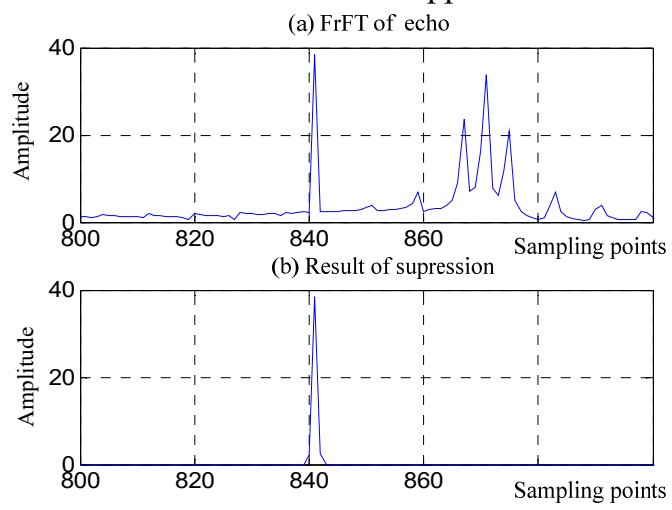

Figure 7. Result of suppression

\subsection{ISRRJ}

A radar pulse is sampled 4 times, and forwarded 3 times at once. The sampling pulse width is $3.25 \mu \mathrm{s}$, the sampling repetition period is $15 \mu \mathrm{s}$, and the JSR is 12 dB.

Different with ISDRJ, ISRRJ is more widely distributed in the FrFT domain and has some characteristics of suppressing interference. The FrFT results of mixed signals are shown in Fig.8 (a). The effect on this interference is shown in Fig.8 (b). Under the condition of high JSR, the target signal can be separated from the ISRRJ, and the CFAR detection is carried out after narrowband filtering.

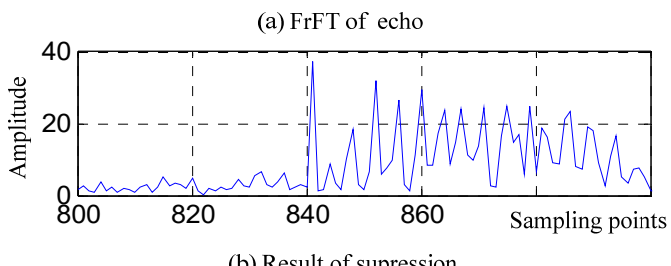

(b) Result of supression

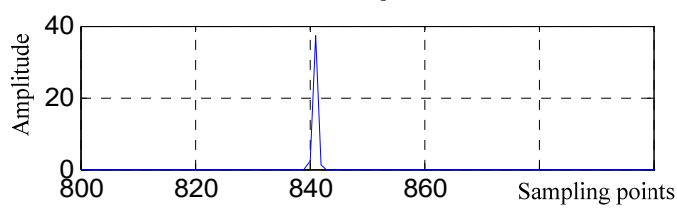

Figure 8. Result of suppression

\subsection{ISCRJ}

A radar pulse is sampled 4 times, and repeat several samples before. The sampling pulse duration is $3.25 \mu \mathrm{s}$, the sampling repetition period is $15 \mu \mathrm{s}$, and the JSR is $15 \mathrm{~dB}$.

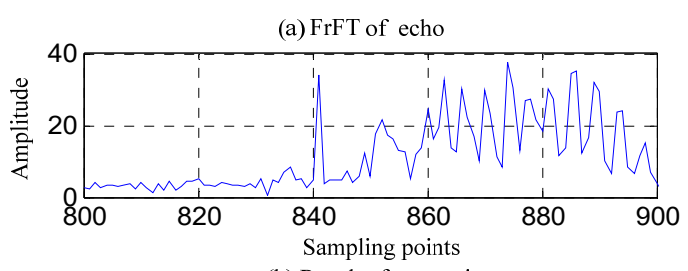

(b) Result of supression

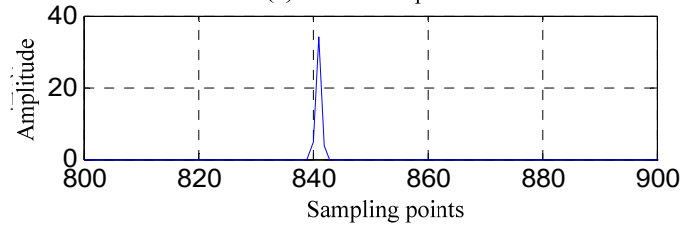

Figure 9. Result of suppression

The FrFT results of ISCRJ are relatively more complicated (shown in Figure 9 (a)), and the results of target echoes can be obtained after interference suppression (shown in Figure 9 (b)).

The suppression effect is closely related to the JSR. Threshold detection is used, and the false alarm probability in the three interference modes varies with the JSR, as shown in Fig.10 (a).

When the JSR is less than $28 \mathrm{~dB}$, the method is available and the false alarm rate is low; when JSR is higher than $33 \mathrm{~dB}$, the method of failure.

In addition, interference suppression is also related to noise, and Fig.10 (b) is the effect of noise on the detection performance of this method, the JSR is $6 \mathrm{~dB}$. When the SNR is not less than $-22 \mathrm{~dB}$, this method can keep very low false alarm probability. Therefore, the proposed method can work under relatively low SNR conditions.
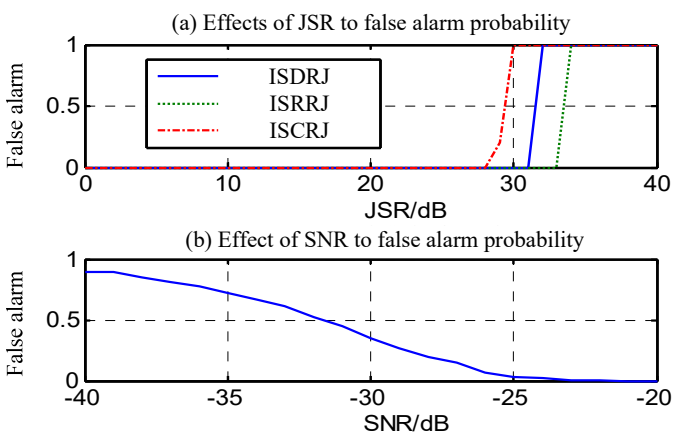

Figure 10. Performance of countermeasure

\section{Conclusion}

In this paper, the interference principles of ISDRJ, ISRRJ and ISCRJ were discussed; the effect of LFM signal after the best order FrFT transform processing was analyzed; The countermeasure of ISRJ for LFM radars based on FrFT has been put forward, and a simulation about it was carried out. The simulation results show that the method is effective for the suppression of LFM ISRJ under the condition of low SNR or high JSR environment.

\section{References}

1. $\mathrm{C} \mathrm{Li}, \mathrm{W} \mathrm{Su}, \mathrm{H} \mathrm{Gu}$, et al. Improved interrupted sampling repeater jamming based on DRFM[C]// 
Signal Processing, Communications and Computing (ICSPCC), 2014 IEEE International Conference, 2014:254-257.

2. D J Feng, $\mathrm{H}$ M Tao, $\mathrm{Y}$ Yang, et al. Jamming de-chirping radar using interrupted-sampling repeater[J]. Science China Information Sciences, 2011, 54(10):2138-2146.

3. X S Wang, J C Liu, W M Zhang, et al. Mathematic principles of interrupted-sampling repeater jamming (ISRJ)[J]. Science in China Series F: Information Sciences, 2007, 50(1):113-123.

4. A K Singh, R Saxena. On convolution and product theorems for FRFT[J]. Wireless Personal Communications, 2012, 65(1):189-201.

5. G C Sun, M Xing, X G Xia, et al. A unified focusing algorithm for several modes of SAR based on FrFT[J]. IEEE Transactions on Geoscience and Remote Sensing, 2013, 51(5):3139-3155.

6. X Chen, J Guan, Z Bao, et al. Detection and extraction of target with micromotion in spiky sea clutter via short-time fractional Fourier transform[J]. IEEE Transactions on Geoscience and Remote Sensing, 2014, 52(2):1002-1018.

7. J Zheng, Z Wang. ICI analysis for FRFT-OFDM systems to frequency offset in time-frequency selective fading channels[J]. IEEE Communications letters, 2010, 14(10):888-890.

8. Z LIU, X WANG, J LIU, et al. Jamming Technique of Interrupted-sampling and Periodic Repeater Based on Digital Radio Frequency Memory[J]. Acta Armamentarii, 2008, 4:006.

9. R Saxena, K Singh. Fractional Fourier transform:A novel tool for signal processing[J]. Journal of the Indian Institute of Science, 2013, 85(1):11.

10. S Liu, T Shan, R Tao, et al. Sparse discrete fractional Fourier transform and its applications[J]. IEEE Transactions on Signal Processing, 2014, 62(24):6582-6595. 